\title{
Desenvolvimento "in vitro" de larvas e juvenis de Emerita brasiliensis Schmitt (Crustacea, Decapoda, Hippidae) sob diferentes condições de temperatura, salinidade e regime alimentar
}

\author{
Ana Clara P. Otegui \& Abilio Soares-Gomes \\ Departamento de Biologia Marinha, Universidade Federal Fluminense. Caixa Postal 100644, 24001-970 Niterói, Rio de \\ Janeiro, Brasil. E-mail: abiliosg@vm.uff.br
}

\begin{abstract}
In vitro" development of larvae and juveniles of the sand crab Emerita brasiliensis Schmitt (Crustacea, Decapoda, Hippidae) under different conditions of temperature, salinity and food diet. Hatched larvae and field sampled juveniles of the sand crab Emerita brasiliensis were reared at different temperatures and food diet in order to evaluate the best conditions for laboratory maintenance. The effects of frequency of changing the aquarium seawater on larval development was also studied evaluating the survival, growth and duration of larval stages. The duration of larval stage and growth of the last three zoes phases correlate positively with temperature. Temperatures between 18 and $26^{\circ} \mathrm{C}$ did not influence the larval survival. The kind of food and frequency of water exchange did not influence the development, growth, and survival of larvae, as well as the development, and survival of juveniles.
\end{abstract}

KEY WORDS. Intertidal; larval development; macrobenthos; marine invertebrates; sandy beach.

RESUMO. Larvas eclodidas em laboratório e juvenis coletados em campo do tatuí Emerita brasiliensis (Hippidae) foram cultivados em diferentes temperaturas e regime alimentar com a finalidade de avaliar as melhores condições para a manutenção da espécie em laboratório. $O$ efeito da frequiência da substituição da água de cultivo sobre o desenvolvimento larvar também foi estudado avaliando-se a sobrevivência, o crescimento e a duração dos estádios larvares. A duração das fases larvares e o crescimento das três últimas fases de zoea correlacionaramse positivamente com a temperatura. As temperaturas testadas, entre 18 e $26^{\circ} \mathrm{C}$ não influenciaram a sobrevivência das larvas. Não houve influencia da alimentação e nem da troca de água no desenvolvimento, crescimento e sobrevivência das larvas, bem como das dietas de microalgas na sobrevivência e desenvolvimento dos juvenis. PALAVRAS-CHAVE. Desenvolvimento larvar; invertebrados marinhos; macrobentos; praias arenosas; tatuí.

O crustáceo Emerita brasiliensis Schmitt, 1935, Hippidae, possui uma ampla distribuição geográfica, ocorrendo em praias arenosas refletivas e dissipativas ao longo da costa oeste do Atlântico, desde o México até o Uruguai (Tam et al. 1996, Calado 1999).

Denominado vulgarmente de tatuí ou tatuíra, é um dos principais representantes da macrofauna das praias arenosas do Rio de Janeiro, ocorrendo em densas populações e apresentando elevada produção secundária, principalmente durante a primavera e o verão (Veloso et al. 1997, 2003). Segundo Veloso \& Cardoso (1999), as flutuações da densidade populacional no Rio de Janeiro estão relacionadas às variações na taxa de recrutamento ao longo do ano, apesar da espécie apresentar reprodução contínua (Veloso et al. 1995). O estudo de populações no sul do Brasil, por sua vez, mostrou uma interrupção no ciclo reprodutivo, associada possivelmente a períodos de baixa temperatura (Veloso \& VALENTIN 1993). Além disso, podem ocorrer variações na história de vida de um local para outro, em função de diferenças na morfodinâmica das praias e outras va- riáveis ambientais (CARDOSO et al. 2003).

A espécie tem desenvolvimento indireto planctônico com duração de dois a três meses, passando por oito ou nove estágios larvares de zoea, após o qual sofrem metamorfose para megalopa e assentam no substrato (Veloso \& Calazans 1992).

Muito embora o desenvolvimento in vitro e através de amostras do plâncton, de E. brasiliensis tenha sido descrito (Veloso \& Calazans 1992), até o momento não foram determinadas experimentalmente as condições ambientais ótimas para o desenvolvimento da espécie.

O objetivo geral foi testar diferentes condições de cultivo de larvas e juvenis de E. brasiliensis em laboratório.

\section{MATERIAL E MÉTODOS}

\section{Cultivo das larvas}

Exemplares de fêmeas ovígeras de E. brasiliensis foram coletados manualmente em 28.III.2001 na Praia de Tucuns, litoral norte do Estado do Rio de Janeiro ( $\left.22^{\circ} 49^{\prime} \mathrm{S}, 41^{\circ} 56^{\prime} \mathrm{W}\right)$. Os 
exemplares foram transportados para o laboratório em caixas isotérmicas e transferidos para aquários, onde diariamente foram observados até a liberação natural das larvas. Durante esse período os indivíduos foram alimentados com uma mistura de microalgas composta pelas diatomáceas Chaetoceros muelleri Lemmerman, 1898 e Skeletonema costatum (Greville) Cleve, 1873 e pela clorofícea Tetraselmis gracilis (Kylin) Butcher, 1956.

A água do aquário e dos béqueres de cultivo foi monitorada quanto ao oxigênio dissolvido, utilizando um oxímetro Digimed, o pH com um pHmetro Analyser, a salinidade com um refratômetro Shibuya e a temperatura da água com um termômetro de coluna.

As larvas recém eclodidas que apresentavam mobilidade normal foram selecionadas e transferidas para béqueres de um litro. Cada béquer recebeu 10 larvas no início do experimento, representando uma densidade de um indivíduo por $50 \mathrm{ml}$ de água. Todos os experimentos foram realizados em triplicata, sendo que os indivíduos mortos foram substituídos de modo a manter uma densidade de 10 indivíduos por réplica.

Os experimentos de temperatura foram realizados em câmaras incubadoras com controle de temperatura e fotoperíodo de 12 horas de luz e 12 horas de escuro. As seguintes faixas de temperatura foram testadas: $18-20^{\circ} \mathrm{C}, 20-22^{\circ} \mathrm{C}, 22-$ $24^{\circ} \mathrm{C}, 24-26^{\circ} \mathrm{C}$.

Quanto à alimentação foram testados naúplios de Artemia franciscana Kellog, 1906 (Artemiidae) com um dia de vida (alimento 1) e naúplios de Artemia franciscana, de um dia de vida, enriquecidos com óleo de peixe (alimento 2). Diariamente foram ofertados 60 naúplios para cada larva. Para obtenção dos naúplios procedeu-se a descapsulação dos cistos de resistência com hipoclorito de sódio durante 10 minutos. O enriquecimento dos naúplios foi obtido através da imersão dos mesmos numa emulsão de água/óleo, preparada adicionando-se 0,1 ml de óleo de peixe "Orient Mix" a $100 \mathrm{ml}$ de água do mar. Também foram testados os efeitos da renovação de água de cultivo, trocando-se a água duas ou três vezes por semana. A tabela I apresenta uma sinopse dos experimentos realizados.

Diariamente os béqueres foram observados e as exúvias e os animais mortos foram recolhidos para verificação dos estádios larvares, segundo Veloso \& Calazans (1992), e medição da largura do telson, que, de acordo com EFFord (1970), representa um bom indicador do tamanho corpóreo das larvas.

\section{Cultivo dos juvenis}

Juvenis e megalopas de E. brasiliensis foram coletados manualmente na zona de varrido da Praia de Tucuns e transportados em caixas isotérmicas para o laboratório. Após um período de três dias de aclimatação em bandejas de plásticos com água do mar, sedimento e aeração constante, foram cultivados durante 30 dias testando-se as seguintes microalgas como alimento: Chaetoceros muelleri, Skeletonema costatum e Tetraselmis gracilis. Os experimentos de alimentação foram realizados com dez indivíduos com comprimento do cefalotórax inferior a $10 \mathrm{~mm}$, mantidos em béqueres de um litro com água do mar
Tabela I. Sinopse dos experimentos realizados com as larvas de $E$. brasiliensis. (Alimento 1) naúplios de Artemia sp.; (alimento 2) naúplios de Artemia sp. enriquecidos com óleo de peixe.

\begin{tabular}{lccc}
\hline Tratamento & Temperatura $\left({ }^{\circ} \mathrm{C}\right)$ & Troca de Água & Alimento \\
\hline T1 & $18-20$ & 3 & 1 \\
T2 & $18-20$ & 2 & 2 \\
T3 & $18-20$ & 3 & 2 \\
T4 & $18-20$ & 2 & 1 \\
T5 & $20-22$ & 2 & 1 \\
T6 & $20-22$ & 3 & 1 \\
T7 & $22-24$ & 2 & 1 \\
T8 & $22-24$ & 3 & 1 \\
T9 & $24-26$ & 3 & 1 \\
T10 & $24-26$ & 2 & 2 \\
T11 & $24-26$ & 3 & 2 \\
T12 & $24-26$ & 2 & 1 \\
\hline
\end{tabular}

filtrada em malha de $0,45 \mu \mathrm{m}$ e uma camada de $2 \mathrm{~cm}$ de sedimento autoclavado. Os béqueres foram mantidos com aeração constante e a água do mar e o sedimento foi renovado duas vezes por semana. Durante a troca de água foram analisados a concentração de oxigênio dissolvido, o pH, a salinidade e a temperatura da água com os mesmos métodos utilizados nos experimentos com as larvas.

Os experimentos foram realizados com cinco réplicas por tratamento, com uma densidade constante de um indivíduo por $100 \mathrm{ml}$ de água. O alimento foi oferecido aos animais três vezes por semana, logo após a renovação da água e sedimento, adicionando-se um volume de microalgas determinado através da contagem de células em câmaras Neubauer e Fuchs Rosenthal.

Diariamente as réplicas foram observadas e as exúvias e os animais mortos foram recolhidos para medição do comprimento do cefalotórax. A biomassa úmida de cada animal foi determinada com uma balança analítica e as taxas de muda e de sobrevivência foram determinadas por contagem direta.

A qualidade nutricional das microalgas utilizadas na alimentação foi determinada através da análise dos teores de proteínas e carboidratos totais, de acordo com os métodos descritos por Lowry et al. (1951) e MykLestad \& HAUG (1972), respectivamente.

\section{Análise dos dados}

Os dados de cada experimento foram analisados através da Análise de Variância por postos de Kruskal-Wallis, utilizando-se um nível de significância de 0,5\%.

A relação entre a temperatura e a sobrevivência dos animais, duração das fases larvares e largura do telson foram determinadas através da correlação não-paramétrica de Spearman.

\section{RESULTADOS}

A partir da coleta de 28.III.2001 ocorreram onze eventos de liberação de larvas, durante um intervalo de aproximada- 
Tabela II. Valores médios de oxigênio dissolvido (OD), pH, salinidade e temperatura da água de manutenção das fêmeas ovígeras e da água dos tratamentos. A temperatura dos experimentos foi mantida sob controle, conforme a tabela I.

\begin{tabular}{lcccc}
\hline & $\mathrm{OD}\left(\mathrm{mg} \mathrm{O} 2 \cdot \mathrm{L}^{-1}\right)$ & $\mathrm{pH}$ & Salinidade & Temperatura $\left({ }^{\circ} \mathrm{C}\right)$ \\
\hline Manutenção & $5,65 \pm 0,19$ & $7,96 \pm 0,14$ & $36,3 \pm 1,3$ & $21,58 \pm 1,02$ \\
Experimentos & $4,70 \pm 0,28$ & $8,03 \pm 0,06$ & $34,00 \pm 0$ & controlada \\
\hline
\end{tabular}

mente dois meses. As condições da água de manutenção são apresentadas na tabela II.

A sobrevivência relativa das diferentes fases larvares não apresentou correlação significativa com a temperatura (Tab. III). A duração das fases larvares, por outro lado, apresentou correlação negativa altamente significativa com a temperatura da água (Tab. IV).

Tabela III. Coeficiente de correlação de Spearman entre a temperatura e a sobrevivência relativa das fases larvares. (ns) Não significativo, (N) número de observações.

\begin{tabular}{ccc}
\hline Fases Larvares & Correlação de Spearman & $\mathrm{N}$ \\
\hline I & $0,02 \mathrm{~ns}$ & 39 \\
II - III & $-0,11 \mathrm{~ns}$ & 39 \\
IV - VIII & $-0,02 \mathrm{~ns}$ & 39 \\
\hline
\end{tabular}

Tabela IV. Coeficientes de correlação de Spearman entre a temperatura da água e o tempo de duração de cada fase larvar. (N) Número de observações, $\left({ }^{* *}\right) p<0,001$.

\begin{tabular}{ccc}
\hline Fases Larvares & Correlação de Spearman & $\mathrm{N}$ \\
\hline I & $-0,63^{* *}$ & 159 \\
II & $-0,81^{* *}$ & 155 \\
III & $-0,87^{* *}$ & 186 \\
IV & $-0,67^{* *}$ & 183 \\
V & $-0,72^{* *}$ & 116 \\
VI & $-0,60^{* *}$ & 90 \\
VII & $-0,57^{* *}$ & 108 \\
VIII & $-0,70^{* *}$ & 101 \\
\hline
\end{tabular}

De modo geral, a taxa de sobrevivência relativa apresentou grande variação entre as réplicas dos experimentos, chegando a $141 \%$ nos tratamentos T3 (estágio VIII) e T10 (estágio VI). Contudo, esta tendência não foi observada nas fases II e III, nas quais a variação foi na maioria das vezes menor que $10 \%$, com exceção do tratamento T2 (fases II e III). As maiores taxas de sobrevivência relativa foram observadas nas fases II e III, variando entre 81 e $100 \%$. As porcentagens de sobrevivência nos tratamentos T7 e T8 foram significativamente maiores que nos tratamentos T1, T3, T4 e T9 da fase IV, que os tratamentos T1, T3 e T5 da fase V e T2 da fase VI (Tab. V).
A duração média do estágio larvar variou entre 49 dias, na faixa de temperatura de 24 a $26^{\circ} \mathrm{C}$, e 90 dias, na faixa de temperatura de 18 a $20^{\circ} \mathrm{C}$.

Quanto à relação entre o tamanho das larvas (aferido pela largura do telson) e a temperatura, foi observada uma correlação negativa para as fases VI e VII (Tab. VI).

A sobrevivência dos juvenis obtidos no laboratório, observada até o décimo dia de cultivo, variou entre 90 a 100\% e, até o trigésimo dia, entre 50 a 54\%. A taxa de sobrevivência, a taxa de muda, o comprimento e a biomassa dos juvenis não variaram significativamente em função das diferentes algas utilizadas na dieta alimentar (Tab. VII).

Quanto ao teor de proteínas das algas utilizadas para alimentar os juvenis, Tetraselmis gracilis apresentou concentrações dez vezes mais elevada que Skeletonema costatum e 3 vezes mais elevada que Chaetoceros muelleri. As concentrações de carboidratos foram uma ordem de grandeza maior em T. gracilis que em S. costatum e C. muelleri (Tab. VIII).

\section{DISCUSÃO}

A correlação negativa observada entre a temperatura e a duração da fase larvar de E. brasiliensis já foi reportada na literatura para outros invertebrados marinhos. As larvas do molusco bivalve Macoma balthica, por exemplo, apresentaram um $\mathrm{Q}_{10}$ (aumento da taxa de desenvolvimento causada pelo aumento da temperatura) de 1,4 (Drent 2002), o que, segundo o autor, representa um desenvolvimento dependente da temperatura. No presente trabalho, a $\mathrm{Q}_{10}$ calculada para temperaturas entre 18 a $26^{\circ} \mathrm{C}$, foi também 1,4 , podendo significar uma igual dependência da temperatura no desenvolvimento da espécie.

Hanson (1969) e Fusaro (1980) sugeriram uma influência similar da temperatura no desenvolvimento dos hipídeos, tendo, entretanto, comparado diferentes espécies, cada qual submetidas a temperaturas distintas. Neste trabalho, as ecdises ocorreram em intervalos menores quando comparado com trabalhos cujo cultivo foi realizado com variações de 5 e $9^{\circ} \mathrm{C}$ (DiAz \& Costlow 1987). Isto pode indicar que menores variações na temperatura de cultivo levam a uma maior sincronia nas mudas num mesmo estágio, principalmente até a quarta zoea.

Neste trabalho o completo desenvolvimento larvar de $E$. brasiliensis ocorreu após oito ou nove estágios de zoea. Contudo, o nono estágio larvar foi observada apenas nas temperaturas entre 24 e $26^{\circ} \mathrm{C}$. A variação no número de estágios larvares é comum entre os Anomura (Rees 1959, KNight 1967, Hanson 1969, EFFord 1970, Diaz \& Costlow 1987), sendo a temperatura um dos

Revista Brasileira de Zoologia 24 (2): 277-282, junho 2007 
Tabela V. Sobrevivência de indivíduos, em porcentagem, nos distintos estádios larvares, por tratamento. Os valores representam médias de três tratamentos e os respectivos desvios-padrões. A significância refere-se ao resultado do teste de Kruskall-Wallis, sendo: (ns) não significativo, $\left({ }^{* *}\right) \mathrm{p}<0,01$ e $\left(^{*}\right) \mathrm{p}<0,05$.

\begin{tabular}{|c|c|c|c|c|c|c|c|c|c|}
\hline \multirow{2}{*}{ Estádios Larvares } & \multicolumn{9}{|c|}{ Tratamentos } \\
\hline & I & II & III & IV & V & VI & VII & VIII & IX \\
\hline $\mathrm{T} 1$ & $63,3 \pm 17$ & $100 \pm 0$ & $100 \pm 0$ & $41,7 \pm 12$ & $16,7 \pm 24$ & $100 \pm 0$ & $100 \pm 0$ & $0 \pm 0$ & - \\
\hline $\mathrm{T} 2$ & $66,7 \pm 12$ & $86,9 \pm 10$ & $81,1 \pm 16$ & $83,3 \pm 24$ & $94,4 \pm 8$ & $33,3 \pm 25$ & $50,0 \pm 50$ & $66,6 \pm 12$ & - \\
\hline $\mathrm{T} 3$ & $60,0 \pm 21$ & $100 \pm 0$ & $100 \pm 0$ & $70,0 \pm 10$ & $34,4 \pm 12$ & $83,3 \pm 24$ & $100 \pm 0$ & $33,3 \pm 47$ & - \\
\hline $\mathrm{T} 4$ & $86,7 \pm 5$ & $96,3 \pm 5$ & $96,3 \pm 5$ & $29,2 \pm 21$ & $87,5 \pm 12$ & $0 \pm 0$ & - & - & - \\
\hline T5 & $78,0 \pm 19$ & $100 \pm 0$ & $94,0 \pm 5$ & $74,0 \pm 20$ & $55,9 \pm 25$ & $96,0 \pm 9$ & $100 \pm 0$ & $90,0 \pm 14$ & - \\
\hline T6 \# & - & - & - & - & - & - & - & - & - \\
\hline $\mathrm{T7}$ & $48,0 \pm 43$ & $100 \pm 0$ & $100 \pm 0$ & $96,0 \pm 5$ & $92,0 \pm 18$ & $89,3 \pm 14$ & $86,7 \pm 12$ & $97,5 \pm 6$ & - \\
\hline $\mathrm{T} 8$ & $50,0 \pm 21$ & $100 \pm 0$ & $94,0 \pm 9$ & $98,0 \pm 4$ & $96,0 \pm 5$ & $93,6 \pm 10$ & $86,7 \pm 12$ & $94,3 \pm 13$ & - \\
\hline T9 & $70,0 \pm 0$ & $95,8 \pm 6$ & $100 \pm 0$ & $42,1 \pm 34$ & $33,4 \pm 33$ & $0 \pm 0$ & - & - & - \\
\hline $\mathrm{T} 10$ & $66,7 \pm 9$ & $90,3 \pm 7$ & $94,4 \pm 8$ & $85,7 \pm 20$ & $93,3 \pm 9$ & $33,3 \pm 47$ & $75,0 \pm 0$ & $66,3 \pm 0$ & $0 \pm 0$ \\
\hline $\mathrm{T} 11$ & $86,7 \pm 12$ & $96,3 \pm 5$ & $96,7 \pm 5$ & $87,8 \pm 8$ & $61,1 \pm 44$ & $58,8 \pm 21$ & $54,2 \pm 21$ & $0 \pm 0$ & - \\
\hline $\mathrm{T} 12$ & $80,0 \pm 8$ & $92,1 \pm 6$ & $95,2 \pm 7$ & $89,7 \pm 7$ & $75,6 \pm 17$ & $20,8 \pm 21$ & $50,0 \pm 50$ & $100 \pm 0$ & $0 \pm 0$ \\
\hline Significância & ns & ns & ns & ns & ** & * & ns & ns & \\
\hline
\end{tabular}

\# O experimento não foi realizado devido à ocorrência de fungo.

Tabela VI. Duração média, expressa em dias, dos estádios larvares em cada tratamento.

\begin{tabular}{|c|c|c|c|c|c|c|c|c|c|}
\hline \multirow{2}{*}{ Tratamentos } & \multicolumn{9}{|c|}{ Estádios Larvares } \\
\hline & I & II & III & IV & V & VI & VII & VIII & IX \\
\hline $\mathrm{T} 1$ & $10,32 \pm 0,16$ & $15,92 \pm 0,14$ & $22,76 \pm 0,59$ & $34,0 \pm 0,59$ & $44,50 \pm 0,71$ & $55,00 \pm 0,00$ & $69,00 \pm 0,00$ & $76,00 \pm 0,00$ & - \\
\hline $\mathrm{T} 2$ & $10,86 \pm 0,43$ & $16,47 \pm 0,41$ & $23,99 \pm 1,11$ & $33,43 \pm 3,39$ & $46,25 \pm 1,64$ & $55,25 \pm 1,06$ & $68,00 \pm 0,71$ & $90,33 \pm 9,07$ & - \\
\hline $\mathrm{T} 3$ & $10,15 \pm 0,13$ & $15,44 \pm 0,10$ & $23,01 \pm 0,45$ & $29,39 \pm 1,99$ & $46,50 \pm 6,87$ & $55,33 \pm 3,51$ & $68,33 \pm 2,08$ & $89,0 \pm 3,46$ & - \\
\hline $\mathrm{T} 4$ & $10,24 \pm 0,72$ & $15,96 \pm 0,27$ & $22,64 \pm 0,34$ & $31,33 \pm 2,98$ & $45,25 \pm 6,72$ & $50,25 \pm 3,18$ & - & - & - \\
\hline T5 & $11,35 \pm 0,20$ & $15,88 \pm 1,03$ & $20,67 \pm 0,58$ & $29,14 \pm 0,35$ & $34,73 \pm 2,05$ & $40,35 \pm 2,59$ & $51,10 \pm 3,09$ & $66,12 \pm 2,16$ & - \\
\hline T6 * & - & - & - & - & - & - & - & - & - \\
\hline $\mathrm{T7}$ & $7,08 \pm 0,10$ & $11,42 \pm 0,48$ & $15,42 \pm 0,11$ & $21,96 \pm 0,13$ & $27,53 \pm 0,46$ & $34,03 \pm 0,59$ & $39,64 \pm 2,12$ & $55,30 \pm 0,91$ & - \\
\hline $\mathrm{T} 8$ & $7,00 \pm 0,00$ & $11,15 \pm 1,11$ & $16,00 \pm 0,00$ & $21,87 \pm 0,19$ & $27,67 \pm 0,69$ & $33,62 \pm 0,36$ & $39,54 \pm 2,28$ & $56,91 \pm 4,38$ & \\
\hline T9 & $6,83 \pm 0,44$ & $10,83 \pm 0,17$ & $14,84 \pm 0,17$ & $22,67 \pm 1,15$ & $26,87 \pm 3,49$ & - & - & - & \\
\hline $\mathrm{T} 10$ & $6,83 \pm 0,29$ & $10,64 \pm 0,13$ & $15,67 \pm 0,38$ & $21,24 \pm 0,21$ & $25,78 \pm 0,69$ & $29,30 \pm 1,84$ & $37,83 \pm 2,17$ & $49,00 \pm 0,00$ & $65,00 \pm 0$ \\
\hline $\mathrm{T} 11$ & $6,33 \pm 0,29$ & $10,49 \pm 0,10$ & $15,72 \pm 1,7$ & $23,28 \pm 1,44$ & $27,0 \pm 0,94$ & $37,57 \pm 1,74$ & $43,75 \pm 0,35$ & $49,50 \pm 0,00$ & - \\
\hline $\mathrm{T} 12$ & $6,94 \pm 0,42$ & $10,74 \pm 0,02$ & $14,67 \pm 0,14$ & $21,89 \pm 0,59$ & $26,67 \pm 2,31$ & $34,61 \pm 2,11$ & $38,50 \pm 0,00$ & $50,00 \pm 0,00$ & $60,00 \pm 0$ \\
\hline
\end{tabular}

* $\mathrm{O}$ experimento não foi realizado devido à ocorrência de fungo.

Tabela VII. Comprimento do cefalotórax, biomassa, taxa de muda e sobrevivência de juvenis de Emerita brasiliensis cultivados com diferentes espécies de microalgas. (ns) Não significativo

\begin{tabular}{|c|c|c|c|c|c|c|}
\hline \multirow{2}{*}{$\frac{\text { Tratamento }}{\text { Alimento }}$} & \multicolumn{2}{|c|}{ Muda } & \multicolumn{2}{|c|}{ Mortos } & \multicolumn{2}{|c|}{ Sobrevivência (\%) } \\
\hline & Taxa de Muda (\%) & Cefalotórax (mm) & Cefalotórax (mm) & Biomassa (g) & $1^{\circ}-10^{\circ}$ dia & $11^{\circ}-30^{\circ}$ dia \\
\hline C. muelleri & $31,11 \pm 27,7$ & $5,70 \pm 0,22$ & $5,96 \pm 0,29$ & $0,059 \pm 0,011$ & $90,00 \pm 12,25$ & $50,00 \pm 21,21$ \\
\hline S. costatum & $31,11 \pm 14,4$ & $5,74 \pm 0,38$ & $5,94 \pm 0,38$ & $0,047 \pm 0,007$ & $94,00 \pm 5,48$ & $54,00 \pm 15,17$ \\
\hline T. gracilis & $37,78 \pm 16,9$ & $5,99 \pm 0,17$ & $5,87 \pm 0,17$ & $0,053 \pm 0,009$ & $100,00 \pm 0,00$ & $54,00 \pm 23,02$ \\
\hline & ns & ns & ns & ns & ns & ns \\
\hline
\end{tabular}

Revista Brasileira de Zoologia 24 (2): 277-282, junho 2007 
Tabela VIII. Concentração de proteínas e carboidratos das microalgas utulizadas na alimentação de Emerita brasiliensis, em diferentes dias de crescimento. Os valores representam médias de três réplicas e são apresentados em volume ( $\mu \mathrm{g} \cdot \mathrm{ml}-1)$ e em picograma por célula $\left(\mathrm{pg} \cdot \mathrm{cel}^{-1}\right)$.

\begin{tabular}{|c|c|c|c|c|}
\hline \multirow{2}{*}{ Espécies } & \multicolumn{2}{|c|}{ Proteína } & \multicolumn{2}{|c|}{ Carboidrato } \\
\hline & $\mu \mathrm{g} \cdot \mathrm{ml}^{-1}$ & pg.cel-1 & $\mu \mathrm{g} \cdot \mathrm{ml}^{-1}$ & pg.cel ${ }^{-1}$ \\
\hline \multicolumn{5}{|l|}{ C. muelleri } \\
\hline 1 & $37,26 \pm 3,37$ & $27,00 \pm 1,40$ & $13,05 \pm 3,13$ & $9,58 \pm 2,95$ \\
\hline II & $39,07 \pm 1,99$ & $19,45 \pm 0,42$ & $11,60 \pm 0,25$ & $5,79 \pm 0,39$ \\
\hline III & $47,98 \pm 3,42$ & $21,04 \pm 2,81$ & $15,07 \pm 0,78$ & $6,58 \pm 0,12$ \\
\hline \multicolumn{5}{|l|}{ S. costatum } \\
\hline I & $18,92 \pm 0,43$ & $9,27 \pm 0,10$ & $12,08 \pm 0,04$ & $5,92 \pm 0,19$ \\
\hline II & $22,04 \pm 0,57$ & $9,59 \pm 0,69$ & $18,12 \pm 1,22$ & $7,90 \pm 1,05$ \\
\hline III & $26,07 \pm 2,35$ & $10,77 \pm 0,86$ & $21,98 \pm 1,54$ & $9,10 \pm 0,92$ \\
\hline \multicolumn{5}{|l|}{ T. gracilis } \\
\hline I & $30,76 \pm 2,43$ & $88,76 \pm 3,97$ & $19,32 \pm 1,02$ & $55,80 \pm 1,76$ \\
\hline II & $29,65 \pm 0,69$ & $77,56 \pm 5,23$ & $20,16 \pm 0,45$ & $52,73 \pm 3,53$ \\
\hline III & $33,39 \pm 2,43$ & $94,78 \pm 4,75$ & $28,13 \pm 0,87$ & $80,24 \pm 9,24$ \\
\hline
\end{tabular}

fatores ambientais que pode influenciar o desenvolvimento, aumentando ou diminuindo o número de estágios larvares (BOYD \& Johnson 1963, FagetTi \& Campodonico 1971, Rochanaburanon \& Willianson 1976). Segundo Criales \& Anger (1986), condições desfavoráveis tendem a aumentar o número de mudas, podendo ser uma adaptação a modificações ambientais, as quais as larvas estão submetidas durante sua permanência na coluna d'água.

A temperatura também apresentou correlação com o crescimento das larvas de E. brasiliensis. Megalopas de menor tamanho, desenvolvidas a partir de larvas igualmente menores, corresponderam às maiores temperaturas de cultivo $\left(24 \mathrm{a} 26^{\circ} \mathrm{C}\right)$. Este fato, associado à ocorrência de um estágio adicional nos tratamentos realizados com temperaturas mais elevadas, pode significar um mecanismo de retardamento da metamorfose que possibilite atingir um tamanho maior no momento de assentamento no substrato. As diferenças na duração da fase larvar, no número de estágios e no crescimento em função da temperatura corroboram com os estudos de Rees (1959) e Diaz \& Costlow (1987).

Os valores observados para a largura do telson, neste estudo, foram intermediários entre os reportados por Veloso \& Calazans (1993) para larvas provenientes de amostras do plâncton. Essa diferença foi mais acentuada para as zoeas VII e VIII, sendo menores que as larvas do plâncton. ReEs (1959), KNIGHT (1967) e EFFord (1970) observaram diferenças similares para Emerita sp. cultivados e coletados no plâncton, o que sugere um possível efeito de uma dieta deficiente ou estresse às condições de laboratório (Levin \& Creed 1986, Drent 2002). Em um estudo realizado com larvas de peixe foi observado que a metamorfose não ocorreu quando a alimentação fornecida era restrita a Artemia sp., ao passo que a alimentação com amos- tras de zooplâncton forneceu os nutrientes necessários à metamorfose (EFFord 1970).

As maiores taxas de sobrevivência foram observadas nos intervalos de 20 a $24^{\circ} \mathrm{C}$, apesar de nenhuma das temperaturas testadas terem inibido a metamorfose. A taxa de sobrevivência média para os tratamentos T7 e T8 para todo o período larvar foi de $34 \%$, valor próximo às maiores taxas reportadas para o gênero. Diaz \& Costlow (1987) e Rees (1959) obtiveram 44 e 15\% para E. talpoida (Say, 1818), respectivamente, EFFORD (1970) obteve 1\% para E. analoga (Stimpson, 1857) e KNIGHT (1967) obteve 42\% para E. rathbunae Schmitt, 1935. O período de muda foi o mais crítico quanto à mortalidade, tendo sido observado a morte de várias larvas com as exúvias presas à região frontal do novo exoesqueleto, fato este também relatado por ReEs (1959) e EFFord (1970).

Neste estudo não foi observado qualquer influência do tipo de alimento na taxa de sobrevivência, taxa de muda, biomassa e comprimento do cefalotórax dos juvenis de E. brasiliensis obtidos em laboratório. Este resultado sugere que não houve preferência alimentar dos tatuís em relação à forma, tamanho ou mobilidade das microalgas utilizadas no cultivo. Em todos os experimentos foram observadas evidências de alimentação, como o comportamento dos jovens se posicionando para filtrar a água e a presença de fezes.

\section{AGRADECIMENTOS}

A Sergio de O. Lourenço pela cessão das microalgas da Coleção de Microalgas "Elizabeth Aidar", Departamento de Biologia Marinha, Universidade Federal Fluminense e auxílio a respeito das técnicas de contagem e análises bioquímicas.

\section{REFERÊNCIAS BIBLIOGRÁFICAS}

Boyd, C.M. \& M.W. Jonhson. 1963. Variations in the larval development of a decapod crustacean, Pleuroncodes planipes Stimpson (Galatheidae). Biological Bulletin 124: 141-152.

Calado, T.C. 1999. Superfamília Hippoidea (tatuíras, tatuís), p. 406-414. In: L. Buckup, \& G. Bond-BucKup (Eds). Os crustáceos do Rio Grande do Sul. Porto Alegre, Editora da Universidade Federal do Rio Grande do Sul, 503p.

Cardoso, R.S.; V.G. Veloso \& C.H.S. Caetano. 2003. Life history of Emerita brasiliensis (Decapoda: Hippidae) on two beaches with different morphodynamic characteristics. Journal of Coastal Research 35 (SI): 392-401.

Criales, M.M. \& K. Anger. 1986. Experimental studies on larval development of the shrimps Crangon cangron and C. allmani. Helgolander Meeresunters 40: 241-265.

Diaz, H. \& J.D. Costlow. 1987. Seasonal developmental sequences of Emerita talpoida (Say) (Anomura, Hippidae). Journal of Coastal Research 3 (2): 167-177.

Drent, J. 2002. Temperature responses in larvae of Macoma balthica from a northerly and southerly population of the European distribution range. Journal of Experimental Marine Biology and Ecology 275: 117-129. 
EFFoRd, I.E. 1970. Recruitment to sedentary marine population as exemplified by a sand crab, Emerita analoga (Decapoda, Hippidae). Crustaceana 18: 293-308.

Fagetti, E. \& I. Campodonico. 1971. Larval development of the red crab Pleurocodes monodon (decapoda, Anomura: Galatheidae) under laboratory conditions. Marine Biology 8: 70-81.

FusAro, C. 1980. Temperature and egg production by the sand crab Emerita analoga (Stimpson) (Decapoda, Hippidae). Crustaceana 38 (1): 55-60.

Hanson, A.J. 1969. The larval development of the sand crab Hippa cubensis (de Saussure) in the laboratory (Decapoda: Anomura). Crustaceana 16: 143-157.

KNIGHT, M.D. 1967. The larval development of the sand crab Emerita rathbunae Schimitt (Decapoda, Hippidae). Pacific Science 21: 58-76.

Levin, L.A. \& E.L. Creed.1986. Effect of temperature and food availability on reproductive responses of Streblospio benedicti (Polychaete: Spionidae) with planctotrophic or lecitotrophic development. Marine Biology 92: 103-113.

Lowry, O.H.; N.J. Rosebrough; A.L. Farr \& R.L. Randall. 1951. Protein measurement with the folin phenol reagent. Journal of Biology and Chemistry 193: 265-275.

Myklestad, S. \& A. Haug. 1972. Production of carbohydrates by the marine Chatoceros affinis var. Willei (Gran.) Hustedt. Effect of the concentration of nutrients in the culture médium. Journal of Experimental Marine Biology and Ecology 9: 125-136.

ReEs, G.H. 1959. Larval development of the sand crab Emerita talpoida (Say) in the laboratory. Biological Bulletin 117: 356-370.

Recebido em 28.VII.2006; aceito em 25.IV.2007.
Rochanaburanon, T. \& D.I. Willianson. 1976. Laboratory survival of larvae of Palaemon elegans Rathke and other Caridean shrimps in relation to their distribution and ecology. Estuarine, Coastal and Marine Science 4: 83-91.

Veloso, V.G. \& D.K. Calazans. 1992. Descrição dos estágios larvais de Emerita brasiliensis Schmitt, 1935 (Decapoda: Hippidae) obtidos de amostras do plâncton, Rio Grande do Sul. Nerítica 7 (1-2): 133-145.

Veloso, V.G. \& J.L. Valentin. 1993. Larval distribution and seasonal abundance of Emerita brasiliensis Schmitt, 1983 (Decapoda: Hippidae) in southern Brazil. Revista Brasileira de Biologia 51 (1): 131-141.

Veloso, V.G. \& R.S. Cardoso. 1999. Population biology of the mole crab Emerita brasiliensis (Decapoda: Hippidae) at Fora beach, Brazil. Journal of Crustacean Biology 19 (1): 147153.

Veloso, V.G.; R.S. Cardoso \& D.B. Fonseca. 1997. Spatio-temporal characterization of intertidal macrofauna at Praianha beach (Rio de Janeiro State). Oecologia Brasiliensis 3: 213225.

Veloso, V.G.; R.S. Cardoso \& M. Petracco. 2003. Secondary production of the intertidal macrofauna of Prainha Beach, Brazil. Journal of Coastal Research 35 (SI): 385-391.

Veloso, V.G.; R.S. Cardoso; P.M. Castro \& L.F.R. Guterres. 1995. Reprodução contínua de Emerita brasiliensis (Decapoda: Hippidae) no Rio de Janeiro, RJ, Brasil. Publicação Especial do Instituto Oceanográfico 11: 217-222.

Tam,Y.K.; I. Kornfield \& F.P. OJeda. 1996. Divergence and zoogeography of mole crabs, Emerita spp. (decapoda: Hippidae), in the Americas. Marine Biology 125: 489-497. 